\title{
COVID-19 in Cancer Patients: A Case Study in Viet Nam
}

\section{Tuyen Kim Cat Vo', Nhi Ngọc Yen Huynh ${ }^{2}$, Dai Duc Nguyen², Nguyen Ha Ta ${ }^{2}$, Minh Thien Nguyen², Tai Van Nguyen ${ }^{2}$, Huy Thanh Dang ${ }^{2}$, Ngoan Tran LE ${ }^{3,4}$}

${ }^{1}$ Department of Obstetrics and Gynecology, Graduate School, International University of Health and Welfare, Japan. ${ }^{2}$ School of Medicine, International University of Health and Welfare, Japan. ${ }^{3}$ Institute of Research and Development, Duy Tan University, Da Nang, Viet Nam. ${ }^{4}$ Department of Public Health, School of Medicine, International University of Health and Welfare, Japan.

\begin{abstract}
Objectives: The aim was to describe the characteristics and outcomes of treatments for coronavirus infection in cancer survivors in Viet Nam from 23 January to 6 May 2020. Methods: We accessed data registration and publication of cases tested positive with COVID-19 by the Ministry of Health in Viet Nam. Characteristics of reported positive cases included age, sex, sources of infection, nationality, the onset of symptoms, and history of chronic diseases. The outcomes included incubation, suspected, clinical course, and clearance time by the subgroup of patients having a history of cancer and non-cancer. T-test was used to compare two means of these groups. Results: Three men patients were cancer survivors ( $1.1 \%$ of 271 cases), a Chinese business aged 66 having lung cancer (ICD-10: C34), a Sweden tourism aged 64 having blood cancer (ICD-10: C81-C96), and a Vietnamese aged 71 having liver cancer (ICD-10: C22). The remaining 268 patients were free-cancer. The clinical course of three cancer survivors was 17.3 days that were shorter 3.2 days when compared to non-cancer, $p=0.2068$. The estimated suspected time was longer in cancer survivors ( 5.3 days) than non-cancer (1.5 days), $\mathrm{p}=0.0394$. The mean age was older in cancer survivors (67-year-old) than non-cancer (35.5-year-old), $p=0.0003$. Conclusions: Cancer survivors might be at high risk of COVID-19 infection due to their underlying vulnerable health condition related to cancer disease and they need extra care to minimize the risk of infection from coronavirus.
\end{abstract}

Keywords: Coronavirus- COVID-19- cancer- Vietnam

Asian Pac Environ Cancer, 3 (1), 35-40

\section{Introduction}

COVID-19 which originated in Wuhan China has been affecting 212 countries and territories around the world. This pandemic has been placing a considerable threat to global health and is a tremendous challenge for all countries. Until May $13^{\text {rd }} 2020$, there are a total of 4,289,804 confirmed cases consisting of $2,455,823$ active cases and 1,833,261 closed cases, and a death toll of 288,346 [1]. The continuing efforts of the government and health care system in every country is reducing the number of infected cases and mortality. To reduce the mortality rate, it is important to pay special attention to elderly patients and those with underlying diseases who have a higher risk of adverse outcomes. Of note, compared to non-cancer patients, cancer patients
Submission Date: 07/23/2020Ａcceptance Date: 08/01/2020

\footnotetext{
Corresponding Author:

Dr. Ngoan Tran LE

Institute of Research and Development, Duy Tan University, Da Nang, Viet Nam. Department of Public Health, School of Medicine, International

University of Health and Welfare, Japan.

Email: letngoan@hmu.edu.vn
}

showed a higher risk of developing severe events, including death and/or intensive care unit admission, particularly in those who received antitumor treatment within 14 days of Covid-19 diagnosis [2-3]. However, published information regarding the prevalence of Covid-19 infection and clinical presentation among them is limited and inconsistent.

Among countries affected by this pandemic, Vietnam was one of the first countries where the COVID-19 pandemic spread to the first two patients (both Chinese) reported on 23 January [4]. Also, there are several geographical and social factors putting Vietnam in a high risk of Coronavirus effect. Firstly, Vietnam, situated in Southeast Asia (Figure 1), shared long land 
borders to the north and a high volume of trade with China where the pandemic first significantly expanded. With a population of over 97 million in 2020, Vietnam is among the countries with high population density. Given the geographical and demographical features, Vietnam is at a high risk of importing COVID-19 from oversea with the highest number of patients concentrated in Ha Noi and Ho Chi Minh City Secondly, there is a large number of Vietnamese citizens living and studying overseas mainly in Europe, Oceania, and the United States [5], raising the chance of importing COVID-19 patients from oversea as they returned to Vietnam during COVID-19 pandemic.

However, by rigorous risk management of the government and the continuous effort of the health care personnel, Vietnam has been currently recognized to be successful in controlling the disease. Until May 6th, the number of COVID - 19 confirmed cases in Vietnam was 271, in which 232 patients had recovered were released from the hospital, 39 are being isolated for treatment and no deaths recorded [4]. Outstandingly, three patients with cancer history were also treated and discharged from the hospital.

\section{Objectives}

We performed this study to describe the characteristics and outcomes of treatments for coronavirus infection in cancer survivors in Viet Nam from $23^{\text {rd }}$ January to $6^{\text {th }}$ May 2020.

\section{Materials and Methods}

\section{Study design and data resource}

A case study was designed to describe the demographic and clinical characteristics and outcomes of treatments for coronavirus infection in cancer survivors in Viet Nam from $23^{\text {rd }}$ January to 6th May 2020.

Data was extracted from the website of The Ministry of Health in Viet Nam. In the mentioned research period, there were a total of 271 confirmed cases, in which 3 patients had a cancer history. Demographic characteristics of confirmed cases included age, sex, sources of infection, nationality, the onset of symptoms, and history of chronic diseases.

In terms of clinical presentation, incubation time, suspected time, clinical course, and clearance time were investigated by the subgroup of patients having a history of cancer and non-cancer. Incubation time was defined as the time elapsed between exposure to the case confirmed positive with COVID-19 and when symptoms and signs were first apparent. The suspected time was the time from the date of onset of symptoms to the date confirmed positive with the virus. A clinical course was the time from the date of onset of symptoms to the discharge date. A clearance time was the time from the date confirmed positive with COVID-19 to the discharge date.

\section{Data analysis}

Data was reviewed and cross-checked between information sources, cleaned, encoded, and reported by Excel software, analyzed by STATA 14.0. Firstly, descriptive analysis was used to explore the distribution of different variables. Secondly, the difference between the means of two subgroups was identified by using the T-test. All p_values were two-sided, and $\mathrm{p}<0.05$ (alpha value) was considered to indicate statistical significance.

\section{Ethical approval}

Information was uploaded on the website of the Ministry of Health for the community's accessibility to prevent the outbreak of disease. There was no identification information (name, personal address, date of birth) and other private information presented neither on the MOH's webpage nor in the database, consent was therefore legally not required.

\section{Results}

From 23 January 2020 to 6 May 2020 Vietnam, among 271 confirmed cases, three men patients with cancer history ( $1.1 \%$ of confirmed cases) were recorded. Among 271 positive cases, 232 patients were recovered and discharged from hospitals, Figure 2.

Cancer case 1: a Chinese man from Wuhan, China, aged 66 had underlying chronic diseases of lung cancer (ICD-10: C34, completed cancer treatment course), diabetes, inserted three stains in his corona heart. He landed in the Noi Bai International Airport on 13 January 2010, then travel by air to Nha Trang city on 17 January, and took a train to Ho Chi Minh City on 19 January, and the last destination by taxi to the Long An city on 20 January 2020. On 17 January, he reported the onset of symptoms with fever. On 21 January, he visited a health facility at the Ho Chi Minh city and was suspected to be infected by COVID-19. On 22 January, a subsequent test confirmed him as being infected and admitted to Cho Ray Central hospital. On 10 February 2020, a second test for COVID-19 infection conducted showed a preliminary negative result. On 12 February 2020, he was discharged from hospital and his health was recovered. The clinical course was for 26 days, from 17 January to 12 February 2020 and hospitalized time was 21 days.

Cancer case 2: a Sweden tourism aged 64 having

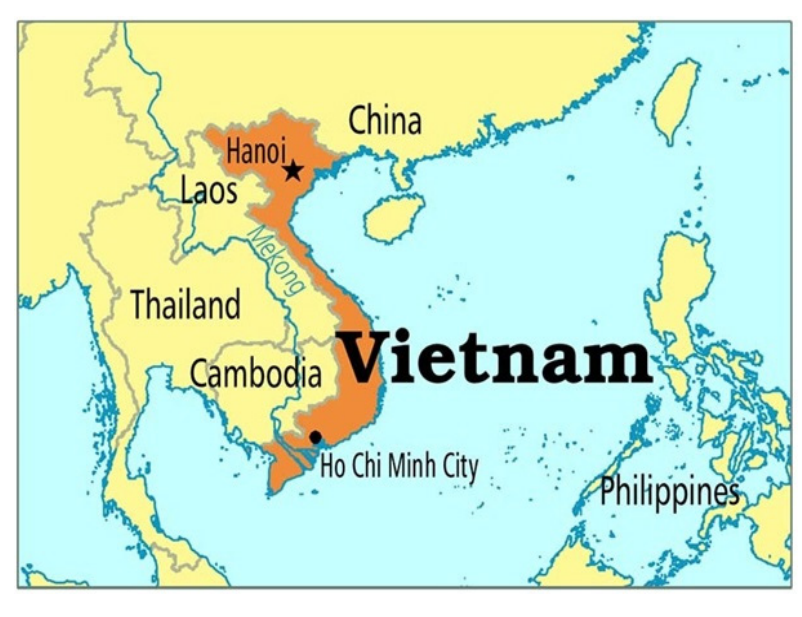

Figure 1. Map of Viet Nam. Source: https://www. itourvn. com/blog/vietnam-s-geography 


\begin{tabular}{|c|c|c|c|c|}
\hline & \multirow[b]{2}{*}{ Non-cancer } & \multirow[b]{2}{*}{ Cancer } & \multicolumn{2}{|c|}{ Total } \\
\hline & & & case & Percent \\
\hline \multicolumn{5}{|l|}{ Age } \\
\hline $0-19$ & 26 & 0 & 26 & 9.6 \\
\hline $20-29$ & 100 & 0 & 100 & 36.9 \\
\hline $30-39$ & 43 & 0 & 43 & 15.9 \\
\hline $40-49$ & 43 & 0 & 43 & 15.9 \\
\hline $50-59$ & 32 & 0 & 32 & 11.8 \\
\hline $60-69$ & 19 & 2 & 21 & 7.7 \\
\hline $70+$ & 5 & 1 & 6 & 2.2 \\
\hline Total & 268 & 3 & 271 & 100 \\
\hline \multicolumn{5}{|c|}{ Source of infection } \\
\hline Import case & 161 & 2 & 163 & 60.1 \\
\hline Local case & 107 & 1 & 108 & 39.9 \\
\hline Total & 268 & 3 & 271 & 100 \\
\hline \multicolumn{5}{|l|}{ Nationality } \\
\hline Vietnamese & 221 & 1 & 222 & 81.9 \\
\hline Foreigner & 47 & 2 & 49 & 18.1 \\
\hline Total & 268 & 3 & 271 & 100 \\
\hline
\end{tabular}

Chronic Myeloid Leukemia (ICD-10: C81-C96) and treated by Nilotimid from the last four years, he arrived in Hanoi in December 2019. Recent 4 months, he skipped taking this medicine during a sightseeing time in Viet Nam. On 26 March, he was suffered from injury and visit a hospital in Hanoi. On 1 April, he visited the National Hospital of Blood Transfusion and Disease due to his nose bleeding. I was suspected of being followed up for COVID-19. On 3 April 2020, a subsequent test confirmed him as being infected and admitted to the National Hospital of Tropical Medicine in Hanoi city. After 7 days, on 10 April, a second test for COVID-19 infection conducted showed a preliminary negative result and discharged from the hospital and transferred to the other health facility for the next 14 days of follow-up. A clinical cause was 10 days, from 1 to 10 April 2020.

Cancer case 3: a Vietnamese aged 71 having liver cancer (ICD-10: C22). On 11 and 19 March 2020, he has visited twice the department of outpatient of the Bach Mai hospital in examining for liver cancer. On 28 March, he reported the onset of fever. On 1 April, he visited Vinh Phuc Provincial Hospital and taking sample secure and the results on the next day showed a positive with COVID-19. After three times of negative results, he was discharged from hospital on 14 April 2020. A clinical cause was 18 days, from 28 March to 14 April 2020.

The remaining 268 patients were reported being free from any cancer form. Among the three cancer patients, there were two imported cases (Foreigners) and one local case (Vietnamese). Their age was 60 or older, Table 1 .

The age (mean \pm standard deviation) was older in cancer survivors (67 \pm 3.6 -year-old) than non-cancer ( 35.5 \pm 15 .6-year-old $)(\mathrm{p}=0.0003)$. The incubation time in cancer patients and non - cancer patients were respectively 7.0 \pm 4.2 days, and $7.8 \pm 6.0$ days. The estimated suspected time was longer in cancer survivors $(5.3 \pm 2.1$ days $)$ than non-cancer $(1.5 \pm 3.7$ days $), p=0.0394$. The clinical course of three cancer survivors was $17.3 \pm 8.5$ days that were shorter 3.2 days when compared to non-cancer $(20.5$ \pm 6.6 days, $\mathrm{p}=0.2068$ ), Table 2 .

\section{Discussions}

The main findings included the outcomes of three cancers patients who were suffered from the deadly coronavirus named COVID-19 in Viet Nam. They were aging persons and had all recovered and discharged from hospitals. The estimated mean of clinical course was 17.3 days, the clearance time was 12.0 days, and the suspected

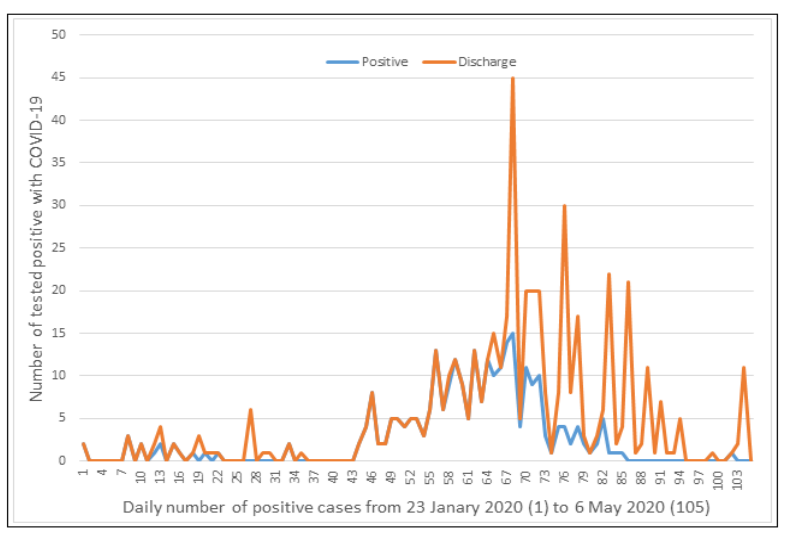

Figure 2. Timeline of the Number of a Tested Positive with COVID-19 and Recovered Cases (Discharge from hospitals) During 105 Continuing Days from 23 January 2020 to 6 May 2020. Total a tested positive with COVID-19: 271; Total recovered cases: 232 by 6 May 2020 6:00 AM (Hanoi time) 
Table 2. The Interim Outcome of Cancer and Non-cancer Management and Ttreatment

\begin{tabular}{|c|c|c|c|c|c|c|c|c|}
\hline \multicolumn{4}{|c|}{ Cases number } & \multicolumn{5}{|c|}{ Quantiles } \\
\hline Variables & (n) & Mean & S.D. & Min & 0.25 & Mdn & 0.75 & $\operatorname{Max}$ \\
\hline \multicolumn{9}{|l|}{ Total of 271 cases } \\
\hline age & 271 & 35.9 & 15.9 & 0.3 & 23 & 30 & 48 & 88 \\
\hline incubation time & 77 & 7.8 & 6 & 0 & 3 & 7 & 12 & 25 \\
\hline suspected time & 271 & 1.6 & 3.7 & 0 & 0 & 0 & 2 & 33 \\
\hline clinical course & 77 & 20.4 & 6.6 & 6 & 16 & 20 & 23 & 43 \\
\hline clearance time & 232 & 17 & 7.5 & 1 & 12 & 16 & 21 & 48 \\
\hline \multicolumn{9}{|l|}{ Cancer patients } \\
\hline Age \& & 3 & 67 & 3.6 & 64 & 64 & 66 & 71 & 71 \\
\hline incubation time & 2 & 7 & 4.2 & 4 & 4 & 7 & 10 & 10 \\
\hline suspected time \$ & 3 & 5.3 & 2.1 & 3 & 3 & 6 & 7 & 7 \\
\hline clinical course \# & 3 & 17.3 & 8.5 & 9 & 9 & 17 & 26 & 26 \\
\hline clearance time & 3 & 12 & 7.2 & 6 & 6 & 10 & 20 & 20 \\
\hline \multicolumn{9}{|l|}{ Non-cancer patients } \\
\hline Age \& & 268 & 35.5 & 15.6 & 0.3 & 23 & 30 & 47 & 88 \\
\hline incubation time & 75 & 7.8 & 6 & 0 & 3 & 7 & 12 & 25 \\
\hline suspected time $\$$ & 268 & 1.5 & 3.7 & 0 & 0 & 0 & 1.5 & 33 \\
\hline clinical course \# & 74 & 20.5 & 6.6 & 6 & 16 & 20 & 23 & 43 \\
\hline clearance time & 229 & 17.1 & 7.5 & 1 & 12 & 16 & 21 & 48 \\
\hline
\end{tabular}

S.D, Standard deviation; Mdn, Median; \$ Suspected time; Cancer vs Non-cancer patients: difference 3.8 day; p,0.0394; \# Clinical time, Cancer vs Non-cancer patients, difference -3.2 day; p,0.2068; \& Age, Cancer vs Non-cancer patients, difference 31.5 day; p,0.0003

time was 5.3 days. These three cancer survivors were not in any ongoing treatment course of either chemotherapy or radiotherapy. There were non-serious complications that occurred among them.

The present findings were consistent with the results in the U.K. Investigators were not able to identify evidence that cancer patients on cytotoxic chemotherapy or other anticancer treatment are at an increased risk of mortality from COVID-19 disease compared with those not on active treatment .

Coronaviruses are enveloped, positive-sense, singlestranded RNA viruses, infecting a wide variety of host species. The novel coronavirus, COVID-19, or 2019$\mathrm{nCoV}$, is named as SARS-CoV-2 due to its high homology $(\sim 80 \%)$ to SARS-CoV [6]. The pathology of this virus is the production of inflammatory cytokines such as IL-1, IL-6, IL-8, and TNF- $\alpha$, which attract alveolar macrophages, neutrophils (IL-8), and induce alveolar and interstitial inflammation [6]. These cytokines, additionally, trigger acute endothelial cell activations, resulting in the expression of tissue factors, activation of the coagulation cascade, and vascular thrombosis [7]. Consequently, patients with immunodeficiency are at a high risk of COVID-19 infection and adverse outcomes with a high occurrence of clinically severe events. Cancer patients are theoretically supposed to be vulnerable to COVID -19 for their systematically immunosuppressive situation due to the malignancy and anticancer treatments. COVID-19 infection in cancer patients is therefore a topic of great interest and needs to be carefully investigated to decrease the mortality in this patient group.

In an article published in The Lancet Oncology, the results showed that $18(1 \% ; 95 \%$ CI $0 \cdot 61-1 \cdot 65)$ of 1590 COVID-19 cases had a history of cancer, which seems to be higher than the incidence of cancer in the overall Chinese population [3]. Nevertheless, the sample size of this study is small and heterogeneous, with variable disease courses and treatments, therefore cannot represent the whole population of cancer patients. Cancer patients in this study also have a higher median age and a longer smoking history, which are both COVID-19 risk factors and indicators of worse outcomes. Another study by L Zhang et al. concluded that COVID-19 infected patients presented poor outcomes with a high occurrence of clinically severe events and mortality [8]. However, this study itself is also based on a small sample size, and some important confounders such as tumor stage were not included. Yong $\mathrm{Hu}$ et al [9]. reviewed 21 studies (including 20 were conducted in China and one in Singapore) reported the percentage of $1.2 \%$ of COVID-19-infected patients with cancer history while these figures in New York City (USA) [10] and Lombardy Region (Italy) [11] were $6 \%$ and $8 \%$ respectively. In one meta-analysis review including 10 studies with 3,403 hospitalized patients, the pooled prevalence of malignancy among hospitalized COVID - 19 patients was estimated to be $0.92 \%$ [12]. In our study, the prevalence of cancer patients among confirmed COVID - 19 patients is $1.1 \%$, which is consistent with these above studies. People with malignant disease history must be well informed about their vulnerable situation and protect themselves by following hygiene guidelines such as regular hand wash, wearing masks, and restricting social interactions. For the health care personnel, it reminds physicians that 
intensive attention should be paid to patients with cancer, in case of rapid deterioration.

The mean age of cancer patients subgroup is higher than non - cancer patients subgroup $(\mathrm{p}=0.0003)$. This result is reasonable since malignant diseases are likely to occur in older people. This increases the risk of adverse outcomes for this group of patients because advanced age is one of the main factors for aggravating the disease [13].

Based on our study, the clinical course of three cancer survivors was 17.3 days that were shorter 3.2 days when compared to non-cancer patients. However, the difference was not statistically significant meaning $(p=0.2068)$. In terms of the suspected time, there is a significant difference between the two subgroups. For details, the cancer patients have long suspected time (5.3 days) comparing with the non - cancer patients. Nevertheless, there are only three cases included in the present study. Further studies, therefore, are warranted to make a conclusive association between COVID-19 and cancer history.

Cancer survivors have a significant long suspected time and they were traveling in community with the onset of symptoms for 6 days (Cancer case 1), 3 days (Cancer case 2), and 6 days (Cancer case 3 ). Before the onset of symptoms, the incubation period was estimated to be 5.2 days. This long delay time from an exposure date to the date of tested positive with COVID-19 might cause a massive spread of the viral infection in communities. That is, the first case has transmitted the disease to the hotel officer at the Khanh Hoa city and transmitted the disease to his son during sharing a hotel room with him there. The cancer case 3 has caused a big cluster of COVID-19 at a commune with 10,878 residents. A total of 13 patients tested positive with COVID-19 who are living in this commune and have rich contact with the cancer case 3 . The commune was being lockdown for 28 days to prevent the viral disease.

\section{Limitation of the study}

The main limitation of this study was the small sample size, which is common in studies of rare diseases. The other limitation was the lack of detailed treatment availability of these patients.

\section{Perspectives}

Although recent studies have improved our knowledge about the novel coronavirus, many questions are still unknown. More research with higher number cases about COVID infection in cancer patients needs to be done to understand more about this virus to effectively interrupt its transmission, to identify in the early stage of the disease, and to ameliorate the current treatment. Comprehensive support and funding from the community, local governments, together with collaborations with international organizations are vital as the war with the virus may last long.

In conclusions, cancer survivors might be at high risk of COVID-19 infection due to their underlying vulnerable health condition related to cancer disease and they need extra care to minimize the risk of infection from coronavirus.

\section{Acknowledgments}

We thank healthcare practitioners of the clinics and hospitals in Vietnam for their hard work of testing, tracing, isolating, and treating activities in controlling the ongoing Coronavirus pandemic in Viet Nam.

\section{Funding}

There was no funding

\section{Conflict of interest}

There are no conflicts to disclose.

\section{References}

1. WHO. Coronavirus disease (COVID - 19) outbreak situation, 2020 [cited 2020 May 15]. https://www.who.int/ emergencies/diseases/novel-coronavirus-2019]..

2. Dai M, Liu D, Liu M, Zhou F, Li G, Chen Z, Zhang Z, You H, Wu M, Zheng Q, Xiong Y, Xiong H, Wang C, Chen C, Xiong F, Zhang Y, Peng Y, Ge S, Zhen B, Yu T, Wang L, Wang H, Liu Y, Chen Y, Mei J, Gao X, Li Z, Gan L, He C, Li Z, Shi Y, Qi Y, Yang J, Tenen DG, Chai L, Mucci LA, Santillana M, Cai H. Patients with cancer appear more vulnerable to SARS-COV-2: a multi-center study during the COVID-19 outbreak. Cancer Discovery. 202004 28;:CD-20-0422. https://doi.org/10.1158/2159-8290.cd-20-0422

3. Liang W, Guan W, Chen R, Wang W, Li J, Xu K, Li C, Ai Q, Lu W, Liang H, Li S, He J. Cancer patients in SARSCoV-2 infection: a nationwide analysis in China. The Lancet Oncology. 2020 03;21(3):335-337. https://doi.org/10.1016/ s1470-2045(20)30096-6

4. COVID - 19 statistics of Vietnam: Ministry of Health (Vietnam), 2020 [cited 2020 May 15]. https://ncov.moh. gov.vn/].

5. Hue N. 190,000 Vietnamese students abroad are now urged by the Ministry of Education and Training. Tien Phong 2020 [cited 2020 May 16th]. https://www.tienphong.vn/giaoduc/190000-luu-hoc-sinh-viet-nam-dang-o-nuoc-ngoai-bogddt-khuyen-cao-khan-1626494.tpo]..

6. Yuki K, Fujiogi M, Koutsogiannaki S. COVID-19 pathophysiology: A review. Clinical Immunology. 2020 06;215:108427. https://doi.org/10.1016/j.clim.2020.108427

7. McGonagle D, O’Donnell JS, Sharif K, Emery P, Bridgewood C. Immune mechanisms of pulmonary intravascular coagulopathy in COVID-19 pneumonia. The Lancet Rheumatology. 2020 07;2(7):e437-e445. https://doi. org/10.1016/s2665-9913(20)30121-1

8. Zhang L, Zhu F, Xie L, Wang C, Wang J, Chen R, Jia P, Guan H, Peng L, Chen Y, Peng P, Zhang P, Chu Q, Shen Q, Wang Y, Xu S, Zhao J, Zhou M. Clinical characteristics of COVID-19-infected cancer patients: a retrospective case study in three hospitals within Wuhan, China. Annals of Oncology. 2020 07;31(7):894-901. https://doi.org/10.1016/j. annonc.2020.03.296

9. Hu Y, Sun J, Dai Z, Deng H, Li X, Huang Q, Wu Y, Sun $\mathrm{L}, \mathrm{Xu} \mathrm{Y}$. Prevalence and severity of corona virus disease 2019 (COVID-19): A systematic review and meta-analysis. Journal of Clinical Virology. 2020 06;127:104371. https:// doi.org/10.1016/j.jcv.2020.104371

10. Richardson S, Hirsch JS, Narasimhan M, Crawford JM, McGinn T, Davidson KW, Barnaby DP, Becker LB, Chelico 
JD, Cohen SL, Cookingham J, Coppa K, Diefenbach MA, Dominello AJ, Duer-Hefele J, Falzon L, Gitlin J, Hajizadeh N, Harvin TG, Hirschwerk DA, Kim EJ, Kozel ZM, Marrast LM, Mogavero JN, Osorio GA, Qiu M, Zanos TP, . Presenting Characteristics, Comorbidities, and Outcomes Among 5700 Patients Hospitalized With COVID-19 in the New York City Area. JAMA. 202005 26;323(20):2052. https://doi.org/10.1001/jama.2020.6775

11. Grasselli G, Zangrillo A, Zanella A, Antonelli M, Cabrini L, Castelli A, Cereda D, Coluccello A, Foti G, Fumagalli R, Iotti G, Latronico N, Lorini L, Merler S, Natalini G, Piatti A, Ranieri MV, Scandroglio AM, Storti E, Cecconi M, Pesenti A, . Baseline Characteristics and Outcomes of 1591 Patients Infected With SARS-CoV-2 Admitted to ICUs of the Lombardy Region, Italy. JAMA. 202004 28;323(16):1574. https://doi.org/10.1001/jama.2020.5394

12. Emami A, Javanmardi F, Pirbonyeh N, Akbari A. Prevalence of Underlying Diseases in Hospitalized Patients with COVID-19: a Systematic Review and Meta-Analysis. Arch Acad Emerg Med. 2020;8(1):e35.

13. Liu Y, Mao B, Liang S, Yang J, Lu H, Chai Y, Wang L, Zhang L, Li Q, Zhao L, He Y, Gu X, Ji X, Li L, Jie Z, Li Q, Li X, $\mathrm{Lu} \mathrm{H}$, Zhang W, Song Y, Qu J, Xu J. Association between age and clinical characteristics and outcomes of COVID-19. European Respiratory Journal. 202004 20;55(5):2001112. https://doi.org/10.1183/13993003.01112-2020

\section{(i) (8)}

This work is licensed under a Creative Commons AttributionNon Commercial 4.0 International License. 